\title{
Minimizing bias in massive multi-arm observational studies with BCAUS: balancing covariates automatically using supervision
}

\author{
Chinmay Belthangady, Will Stedden and Beau Norgeot ${ }^{*}$
}

\begin{abstract}
Background: Observational studies are increasingly being used to provide supplementary evidence in addition to Randomized Control Trials (RCTs) because they provide a scale and diversity of participants and outcomes that would be infeasible in an RCT. Additionally, they more closely reflect the settings in which the studied interventions will be applied in the future. Well-established propensity-score-based methods exist to overcome the challenges of working with observational data to estimate causal effects. These methods also provide quality assurance diagnostics to evaluate the degree to which bias has been removed and the estimates can be trusted. In large medical datasets it is common to find the same underlying health condition being treated with a variety of distinct drugs or drug combinations. Conventional methods require a manual iterative workflow, making them scale poorly to studies with many intervention arms. In such situations, automated causal inference methods that are compatible with traditional propensity-score-based workflows are highly desirable.
\end{abstract}

Methods: We introduce an automated causal inference method BCAUS, that features a deep-neural-network-based propensity model that is trained with a loss which penalizes both the incorrect prediction of the assigned treatment as well as the degree of imbalance between the inverse probability weighted covariates. The network is trained end-to-end by dynamically adjusting the loss term for each training batch such that the relative contributions from the two loss components are held fixed. Trained BCAUS models can be used in conjunction with traditional propensity-score-based methods to estimate causal treatment effects.

Results: We tested BCAUS on the semi-synthetic Infant Health \& Development Program dataset with a single intervention arm, and a real-world observational study of diabetes interventions with over 100,000 individuals spread across more than a hundred intervention arms. When compared against other recently proposed automated causal inference methods, BCAUS had competitive accuracy for estimating synthetic treatment effects and provided highly concordant estimates on the real-world dataset but was an order-of-magnitude faster.

Conclusions: BCAUS is directly compatible with trusted protocols to estimate treatment effects and diagnose the quality of those estimates, while making the established approaches automatically scalable to an arbitrary number of simultaneous intervention arms without any need for manual iteration.

Keywords: Causal inference, Observational studies, Neural networks, Deep learning

\footnotetext{
* Correspondence: beau.norgeot@anthem.com

Anthem Al, Palo Alto, CA 94301, USA

(c) The Author(s). 2021 Open Access This article is licensed under a Creative Commons Attribution 4.0 International License, which permits use, sharing, adaptation, distribution and reproduction in any medium or format, as long as you give appropriate credit to the original author(s) and the source, provide a link to the Creative Commons licence, and indicate if changes were made. The images or other third party material in this article are included in the article's Creative Commons licence, unless indicated otherwise in a credit line to the material. If material is not included in the article's Creative Commons licence and your intended use is not permitted by statutory regulation or exceeds the permitted use, you will need to obtain permission directly from the copyright holder. To view a copy of this licence, visit http://creativecommons.org/licenses/by/4.0/. The Creative Commons Public Domain Dedication waiver (http://creativecommons.org/publicdomain/zero/1.0/) applies to the data made available in this article, unless otherwise stated in a credit line to the data.
} 


\section{Background}

The ability to identify causal variables and estimate their impact is a critical task across many industrial and scientific fields [1-5]. Causal inferences can be drawn from two, generally distinct, types of analytical methods: randomized experiments and observational studies. Randomized Controlled Trials (RCTs), such as FDA trials to approve new drugs, are the gold standard for causal inference but they are not without shortcomings. Their rigor is often associated with high costs and therefore necessitates relatively small cohorts and short trial durations and may feature surrogate endpoints. Observational studies are increasingly being used to provide supplementary evidence in addition to RCTs $[6,7]$ because they provide a scale and diversity of participants and outcomes that would be infeasible in an RCT. Additionally, observational studies more closely reflect the settings in which the studied interventions will be applied in the future. In many application areas there is therefore increasing interest in using observational studies to complement randomized experiments to determine which actions are likely to produce the most desired outcome for a given circumstance or patient.

While observational studies have advantages, they present a distinct set of challenges. In particular, the effects of interventions may be confounded by variables that affect both treatment assignment as well as treatment outcome. Well-established techniques exist to control for cofounding variables in observational studies and estimate causal treatment effects. The propensity-score method pioneered by Rubin and Rosenbaum [8], and its many extensions such as inverse probability of treatment weighting (IPTW) [9] and Double-Robust (DR) [10] estimation, is widely used in medical research, healthcare, and epidemiology [11]. In addition to providing causal estimates, these methods also provide quality assurance diagnostics that assess balance in covariate distributions between intervention arms. When all potential confounders have been identified, these diagnostics help evaluate the degree to which the estimates can be trusted. However, these methods were developed for studies that involved relatively few simultaneous intervention arms and, being iterative in nature [12], they do not scale well to many modern observational datasets that can involve thousands of simultaneous arms.

In recent years several automated causal inference methods have been reported that demonstrate high accuracy in estimating treatment effects on semi-synthetic datasets where the ground truth is known. Some approaches extend logistic regression models [13, 14] to provide covariate balance. Bayesian Additive Regression Trees [15] (BART) have been used for causal modelling [16] and have demonstrated superior performance on benchmark datasets [17]. With recent interest in using deep neural networks for predictive modelling [18], they have been deployed in the causal inference context as well. Shi et al. [19] and Alaa et al. [20] have used them for joint modeling of propensity scores as well as conditional outcomes. Others have focused on using neural networks to estimate effects of counterfactual treatments without estimating propensity scores [21, 22]. Causal inference using neural-network-based generative models has also been demonstrated [23, 24]. While some of the deep learning architectures mentioned above generate propensity scores $[19,20]$, their use in conjunction with popular techniques like IPTW or DR for computing average treatment effects (ATE) and assessing bias removal has not been demonstrated. Also, the use of these methods in situations where there are a large number of intervention arms has not been explored.

In this study, we developed a method we call Balancing Covariates Automatically Using Supervision (BCAUS) that is directly compatible with trusted protocols to estimate treatment effects and diagnose the quality of those estimates, while making these approaches automatically scalable to an arbitrary number of simultaneous intervention arms without any need for manual iteration. Our approach removes manual iteration through the training of deep neural networks for propensity modelling that explicitly remove covariate imbalance $[19,20]$. This is accomplished by training networks with a joint loss function which penalizes both the incorrect prediction of the assigned treatment as well as the degree of imbalance between inverse probability weighted covariates of intervention arms.

We compared BCAUS with multiple state-of-the-art approaches on two clinical datasets. First, on the Infant Health and Development Program (IHDP), a common public semi-synthetic dataset where the ground truth was known, to assess parity in the accuracy of estimation of the ATE. Second, on a private real-world dataset for Type-2 diabetes, which involved 133 simultaneous intervention arms, to assess scalability. While the ground truth ATEs on the diabetes dataset are unknown, we analyzed similarities between the estimations of BCAUS and BART. We found that BCAUS had competitive accuracy for estimating synthetic treatment effects and provided highly concordant estimates on the real-world dataset but was an order-of-magnitude faster. We see our work as being complementary to other automated approaches while still being compatible with conventional causal inference workflows.

\section{Methods}

The BCAUS method trains a deep neural network with a joint loss term consisting of two components. The first component penalizes the incorrect prediction of treatment assignment as in traditional propensity-score 
models. The second component depends explicitly on the imbalance or bias between intervention arms in the data, forcing the network to learn parameters that minimize this bias. A schematic of our method is shown in Fig. 1. The propensity model is a binary classifier which takes the covariates of each individual as input and predicts whether they have been assigned to the control group or the treatment group. In our work we use deep neural networks with dropout regularization and rectified linear unit (ReLu) activations as the propensity model. The output of the network, $p^{(i)}$ (referred to as a propensity score) is trained against targets 0 (for the control group) or 1 (for the treatment group) using a binary cross entropy loss $\mathcal{L}_{B C E}$. All covariates of each instance are weighted by its inverse probability weight (IPW) and the mean squared error between treatment and control groups is computed to obtain the bias loss
$\mathcal{L}_{\text {BIAS }}$. The losses $\mathcal{L}_{B C E}$ and $\mathcal{L}_{\text {BIAS }}$ can have very different scales and their relative magnitudes may vary batch-tobatch during training. To account for this and to ensure that the contributions of the two components can be precisely tuned, for each training batch we compute a scalar ratio of the losses, $\mu=\mathcal{L}_{B C E} / \mathcal{L}_{B I A S}$ that is detached from the computation graph i.e. gradients are not computed for $\mu$ during backpropagation. The total loss term for training the network is:

$$
\mathcal{L}_{\text {TOTAL }}=\mathcal{L}_{B C E}+v \mu \mathcal{L}_{B I A S}
$$

The hyperparameter $v$ can be adjusted to tune the relative contributions of the two modeling objectives. The cross-entropy loss is computed as:

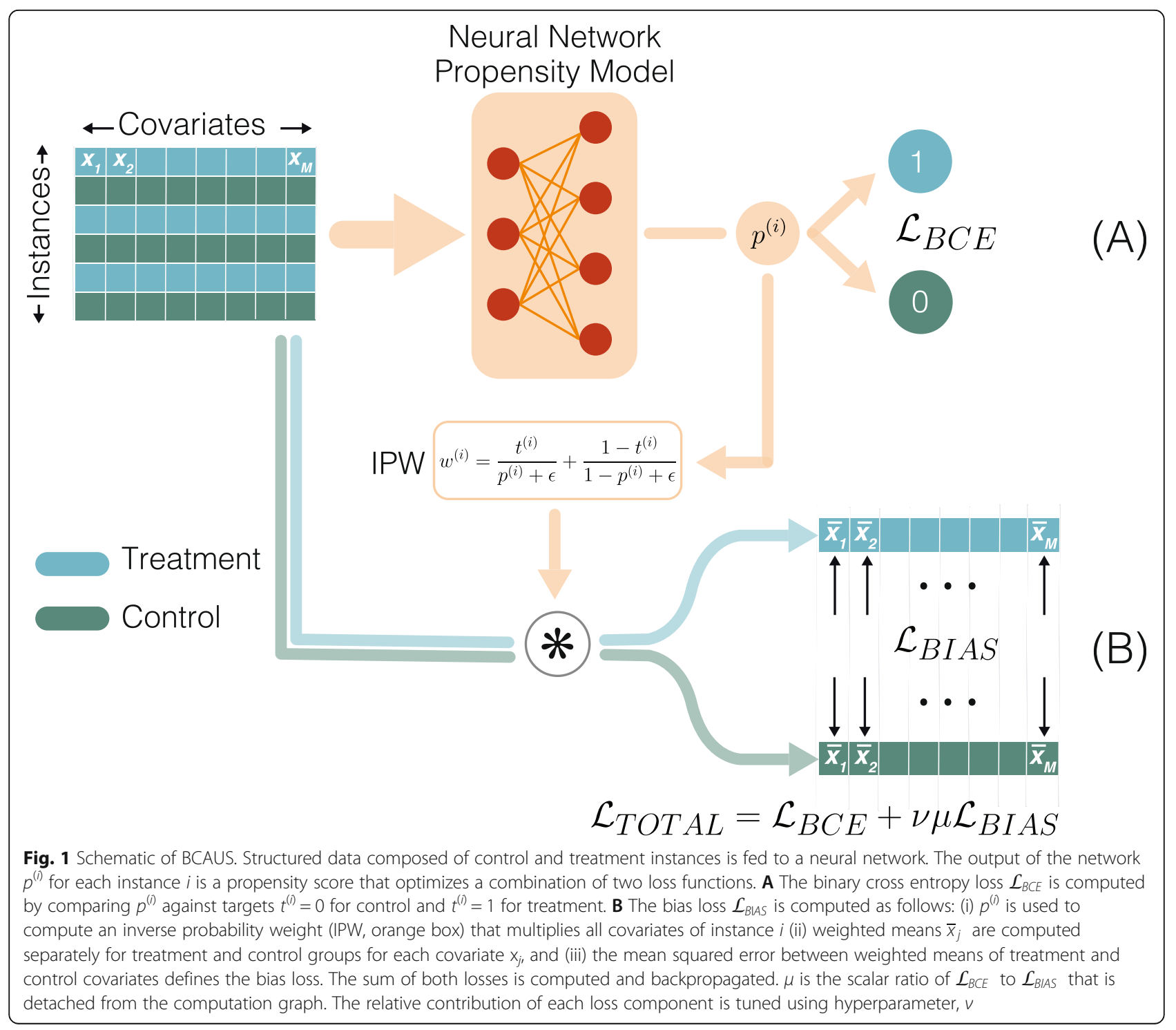




$$
\begin{aligned}
\mathcal{L}_{B C E}= & \sum_{i} t^{(i)} \log \left(p^{(i)}\right) \\
& +\left(1-t^{(i)}\right) \log \left(1-p^{(i)}\right)
\end{aligned}
$$

Here $t^{(i)} \in\{0,1\}$ is the treatment given to individual $i$. To compute the bias loss, the propensity score $p^{(i)}$ is used to compute the IPW:

$$
w^{(i)}=\frac{t^{(i)}}{p^{(i)}+\epsilon}+\frac{1-t^{(i)}}{1-p^{(i)}+\epsilon}
$$

Here $\epsilon$ is a small positive number used to stabilize the denominator. The mean squared error of the $M$ covariates weighted according to Eq.3 is used to calculate the bias loss:

$$
\mathcal{L}_{\text {BIAS }}=\frac{1}{M} \sum_{j=1}^{M}\left(\frac{\sum_{i} t^{(i)} w^{(i)} x_{j}^{(i)}}{\sum_{i} t^{(i)} w^{(i)}}-\frac{\sum_{i}\left(1-t^{(i)}\right) w^{(i)} x_{j}^{(i)}}{\sum_{i}\left(1-t^{(i)}\right) w^{(i)}}\right)^{2}
$$

The two terms in the Eq. 4 represent the weighted means of the covariates for the treatment and control groups respectively. The bias loss $\mathcal{L}_{\text {BIAS }}$ is not calculated against a fixed ground-truth label as is the case for the cross-entropy loss, $\mathcal{L}_{B C E}$. However, being composed entirely of differentiable elements, gradients of this loss can be computed efficiently via backpropagation.

To assess balance, the standardized difference $\Delta_{j}$ for the covariate $x_{j}$ is computed according to:

$$
\Delta_{j}=\frac{\left|\bar{x}_{j, \text { treatment }}-\bar{x}_{j, \text { control }}\right|}{\sqrt{\left(s_{j, \text { treatment }}^{2}+s_{j, \text { control }}^{2}\right) / 2}}
$$

Here $\bar{x}_{j}$ is the weighted mean of $x_{j}$ and $s_{j}^{2}$ is its weighted variance with weights assigned according to Eq. 3. The standardized difference can also be defined for the raw data without the weights, in which case $\bar{x}_{j}$ and $s_{j}^{2}$ represent the unweighted mean and variance respectively.

During training we consider a covariate as being balanced if the standardized difference, $\Delta$ for the weighted covariate is less than 0.1. Several authors have recommended that the standardized difference be used as a diagnostic in clinical observational studies [25-27] with the value of 0.1 being widely accepted as a sufficient threshold for ensuring the removal of bias between treated populations $[28,29]$. We note here that the exact value of this threshold is incidental to our method and any user-specified value may be used; the loss term $\mathcal{L}_{\text {BIAS }}$ tries to explicitly reduce $\Delta$ to 0 for each covariate regardless of this value. We pass the threshold value as a parameter to our training function and use an "earlystopping" strategy where training terminates when $\Delta<$ 0.1 for all covariates. While training may proceed in batches, it is crucial that the number of balanced covariates is always evaluated for the entire dataset.

\section{Results}

We demonstrate the use of BCAUS on two datasets: i) The IHDP dataset with a single pair of control and intervention arms that was introduced by Hill [16] and is used as a benchmark dataset in several recent deeplearning causal inference studies [19-24, 30] and ii) A large observational diabetes dataset studying the effects of anti-hyperglycemic medications on hemoglobin-A1c (HbA1c) values. The ground-truth ATE values for the semi-synthetic IHDP dataset are known. We combined BCAUS with IPTW and DR to estimate ATEs and compared them against the ground-truth. Since the diabetes dataset is drawn from real-word evidence, the groundtruth ATE is unknown. For this reason, we compared ATE values estimated by BCAUS-IPTW against those returned by BART. The choice of BART for comparison was motivated by the fact that it was one of the top performers at the 2016 Atlantic Causal Inference competition [17]. As a diagnostic, we also evaluated the specification of all models by comparing standardized differences $\Delta$ between covariates before and after BCAUS training.

\section{IHDP dataset results}

The IHDP dataset is a modified version of data from a randomized experiment [31] that was conducted to determine the effect of an interventional program on the cognitive development of pre-term babies. The data consists of 25 covariates (labeled $x_{1}$ through $x_{25}$ ) of which 6 are continuous and the rest are binary, a treatment variable, and simulated factual and counterfactual outcomes. The continuous covariates are z-scored to have zero mean and unit variance. By intentionally censoring data from certain subjects, the dataset has been imbalanced to simulate an observational study. To compare results of BCAUS against earlier studies [21-23], we use 1000 realizations of the IHDP dataset made available by Shalit et al. [22]. This dataset was generated by the authors of Ref. 22 using the formulae specified in Ref. 16 such that each realization has a distinct ATE value. While each realization contains a single intervention arm, in aggregate the dataset is analogous to a multi-arm study with 1000 intervention arms. There are 747 individuals in the dataset of whom 608 belong to the control group and 139 belong to the treatment group. The 747 individuals are divided into a training set with 672 individuals and a holdout set of 75 individuals. We subdivided the larger dataset into training (500 samples) and cross-validation 
(172 samples) sets as in other studies [21, 22] but did not perform any additional transformations.

The neural network trained on the IHDP data consisted of two hidden layers with 50 neurons each. The output layer was a single neuron which represented the propensity score. We trained a set of 5 models on a single realization of the IHDP dataset with different values of the hyperparameter $v$ to demonstrate the effect the loss term of Eq. 4 has on achieving covariate balance. The results of our experiments are shown in Fig. 2. In Fig. 2(a), we show the number of balanced covariates as training proceeds for different values of $v$. We observe that when the network is trained with only $\mathcal{L}_{B C E}$ i.e. $v=$ 0 , only a fraction of the covariates are balanced at the end of training. Higher values of $v$ result in faster convergence. The classifier loss $\mathcal{L}_{B C E}$ and the bias loss $\mathcal{L}_{\text {BIAS }}$ are shown in Fig. 2(b) for the model trained with $v=1$. The very different scales of the two losses demonstrates the importance of the parameter $\mu$ in the loss function. Figure 2 (c) shows the standardized difference $\Delta$ between treatment and control, with and without inverse propensity weight (IPW) adjustment, for the model trained with $v=1$. For each of the 25 covariates the adjusted difference lies below 0.1 indicating that the propensity model is well specified. The inset of Fig. 2 (c) shows normalized histograms of the distribution of propensity scores, i.e. output of BCAUS, for treatment and control groups. The large degree of overlap between the distributions is essential for matching-based methods that may be used downstream to estimate the ATE.

To compare against previous work on neural-networkbased causal inference methods, we evaluated BCAUS on 1000 realizations of the IHDP dataset. For each realization we trained BCAUS on 500 training samples and computed the ATE using IPTW and DR. For DR, we trained two linear regressors (for control and treatment respectively) with an L2 penalty and chose the regularization parameter by performing 3 -fold crossvalidation on the training set. We computed the mean absolute error between the estimated and ground-truth ATEs, $\epsilon_{A T E}$ across 1000 realizations and picked BCAUS hyperparameters to minimize this value on 172 crossvalidation samples. Table 1 reports $\epsilon_{A T E}$ between estimated ATEs and ground-truth ATEs. The in-sample $\epsilon_{A T E}$ is computed by combining the 500 training and 172 cross-validation examples and the out-of-sample value is computed on 75 held out examples. We observe that the performance of BCAUS is comparable to other neural-network-based methods and can complement these approaches. We provide code in the Supplementary Information section to reproduce BCAUS results on this data.

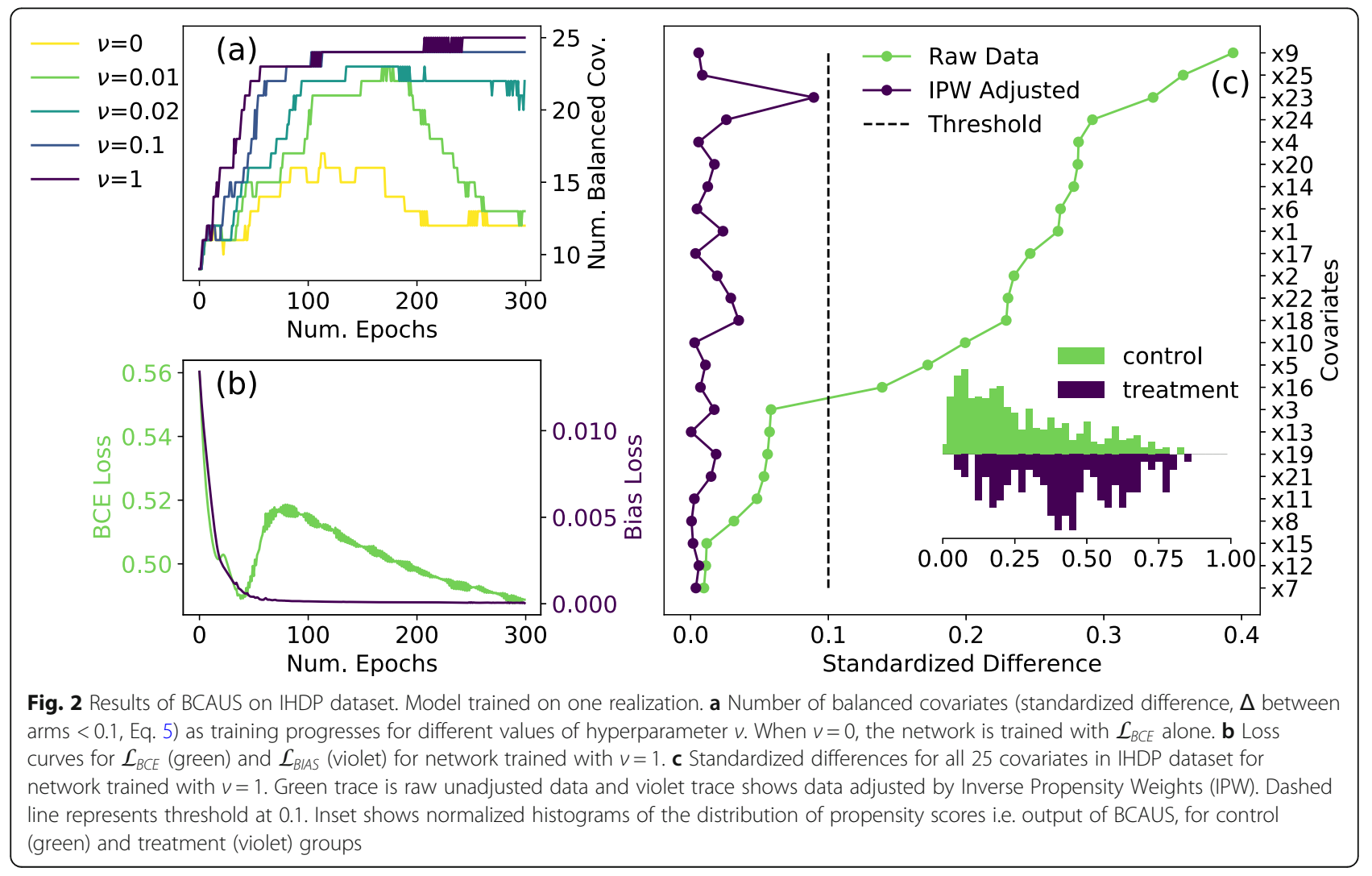


Table 1 Comparing BCAUS against other deep-learning-based causal inference algorithms

\begin{tabular}{lll}
\hline Method & In-sample $\boldsymbol{\epsilon}_{\text {ATE }}$ & Out-of-sample $\boldsymbol{\epsilon}_{\text {ATE }}$ \\
\hline BNN & $0.37 \pm .03$ & $0.42 \pm .03$ \\
BLR & $0.72 \pm .04$ & $0.93 \pm .05$ \\
TARNet & $0.26 \pm .01$ & $0.28 \pm .01$ \\
CFR MMD & $0.30 \pm .01$ & $0.31 \pm .01$ \\
CFR WASS & $0.25 \pm .01$ & $0.27 \pm .01$ \\
GANITE & $0.43 \pm .05$ & $0.49 \pm .05$ \\
Dragonnet & $0.14 \pm .01$ & $0.21 \pm .01$ \\
CEVAE & $0.34 \pm .01$ & $0.46 \pm .02$ \\
BART & $0.47 \pm .02$ & $0.66 \pm .03$ \\
BCAUS IPTW & $0.30 \pm .01$ & $0.60 \pm .02$ \\
BCAUS DR & $0.13 \pm .00$ & $0.29 \pm .01$
\end{tabular}

We include BART for comparison even though it is not neural network based. $\epsilon_{\text {ATE }}$ (lower is better) is the mean absolute error between estimated ATE and ground-truth ATE. BNN Balancing Neural Network [21], BLR Balancing Linear Regression [21], TARNet Treatment-Agnostic Representation Network [22], CFR Counterfactual Regression [22], GANITE Generative Adversarial Nets for inference of Individualized Treatment Effects [24], Dragonnet [19], CEVAE Causal Effect Variational Autoencoder [23], BART Bayesian Additive Regression Trees [16]. In-sample value is computed on 672 examples (training + crossvalidation) and the out-of-sample value is computed on 75 examples in the hold-out set. The standard error across 1000 realizations is reported as the uncertainty. Performance of BCAUS is comparable to other models

\section{Diabetes dataset results}

The diabetes dataset consists of data drawn from health insurance claims of 140,000 patients with Type-2 diabetes mellitus (T2DM) that was collected over a 5-year time period between 1st January 2015 and 31st December 2019. Individuals are included in the dataset if they have a medical claim indicating T2DM (ICD code E11), a history of high levels of glycated hemoglobin (HbA1c > $=9 \%$ ), and prescription claims for anti-hyperglycemic medications. The data tracks the effect of anti-diabetic drugs on HbA1c values and can be used to estimate the comparative effectiveness of individual drugs or combinations of drugs in real-world settings. The data consists of the following 21 covariates: age, gender, 15 variables indicating the presence or absence of comorbid conditions defined by the Charlson Comorbidity Index [32], and 4 variables describing the racial makeup and income levels in the patient's zip code tabulation area (ZCTA). Data on the last four come from US census information [33]. Of these 21 covariates, age, and the four ZCTA variables are continuous while the rest are binary. In addition, a treatment variable denotes which antidiabetic drug or combination was used to treat the individual. The outcome variable is the change in HbA1c value from baseline in a 6-12 month period following treatment. Drugs are identified by their therapeutic class names alone (e.g. GLP-1 Agonists, SGLT2 Inhibitors, etc.). In many instances, patients shift treatment regimens during the 5-year time frame. As a result, in the filtered dataset 289, 000 treatment assignments are observed. Only those treatments where the size of the cohort is greater than 30 are retained. There are 134 unique drug combinations that meet this criterion.

We trained BCAUS on the diabetes dataset setting Insulin (the most common treatment in the dataset) as control and comparing all other drug combinations against this control. We did not explicitly model for time-varying effect but instead considered each treatment-assignment as belonging to a different subject in the study. We used neural networks with two hidden layers of 42 neurons for the BCAUS model. The maximum number of epochs was set to 1000 , but an earlystopping procedure was implemented where training terminated if all 21 covariates remained balanced for more than 10 epochs. The hyperparameter $v$ was set for each control-treatment pair using stratified k-fold crossvalidation, where the network was trained on $\mathrm{k}-1$ folds and the number of balanced covariates (the primary metric to be optimized) were counted for the k-th fold. The number of folds was set to 3 . Once an optimal value of $v$ was obtained, the number of balanced covariates was counted on the entire dataset combing all folds.

In real-word settings such as this, it is not uncommon to observe extreme differences between treatment and control cohort sizes. In our example, the Insulin-treated control group had a cohort size of 44,600 , while the median cohort size of the 133 treatment groups was 163 . From a machine learning perspective, such class imbalances in binary classification tasks are usually treated by sub-sampling or by loss weighting. In experiments described below, we do not use either of these techniques. Training with the joint loss of Eq. 1 is observed to adequately correct for class imbalance. As an example of a treatment cohort with large class imbalance, we consider treatment by a combination therapy of Insulin + Metformin + GLP-1 Agonist + SGLT2 Inhibitor + Sulfonyurea. In this case there were only 125 examples in the treatment cohort, representing a treatment-to-control cohort size ratio of approximately 1:350. As shown in Fig. 3(a) BCAUS was able to remove covariate imbalance present in the unadjusted raw data. The inset of Fig. 3(a) shows normalized histograms of the distribution of propensity scores. To measure the performance of BCAUS on all 133 arms, we count the number of covariates which have low bias $(\Delta<0.1)$ for each arm in the raw data prior to BCAUS training and adjustment. A histogram is shown in Fig. 3(b). We see that in none of the 133 arms are all 21 covariates balanced. The median number of balanced covariates is 9. In Fig. 3(c), we show the same histogram but after BCAUS training and adjustment. In a majority of cases (124 of 133) all 21 covariates are balanced. For a small number of intervention arms (8 of 133) BCAUS is able to balance $\geq 18$ covariates but not all 21 . These 

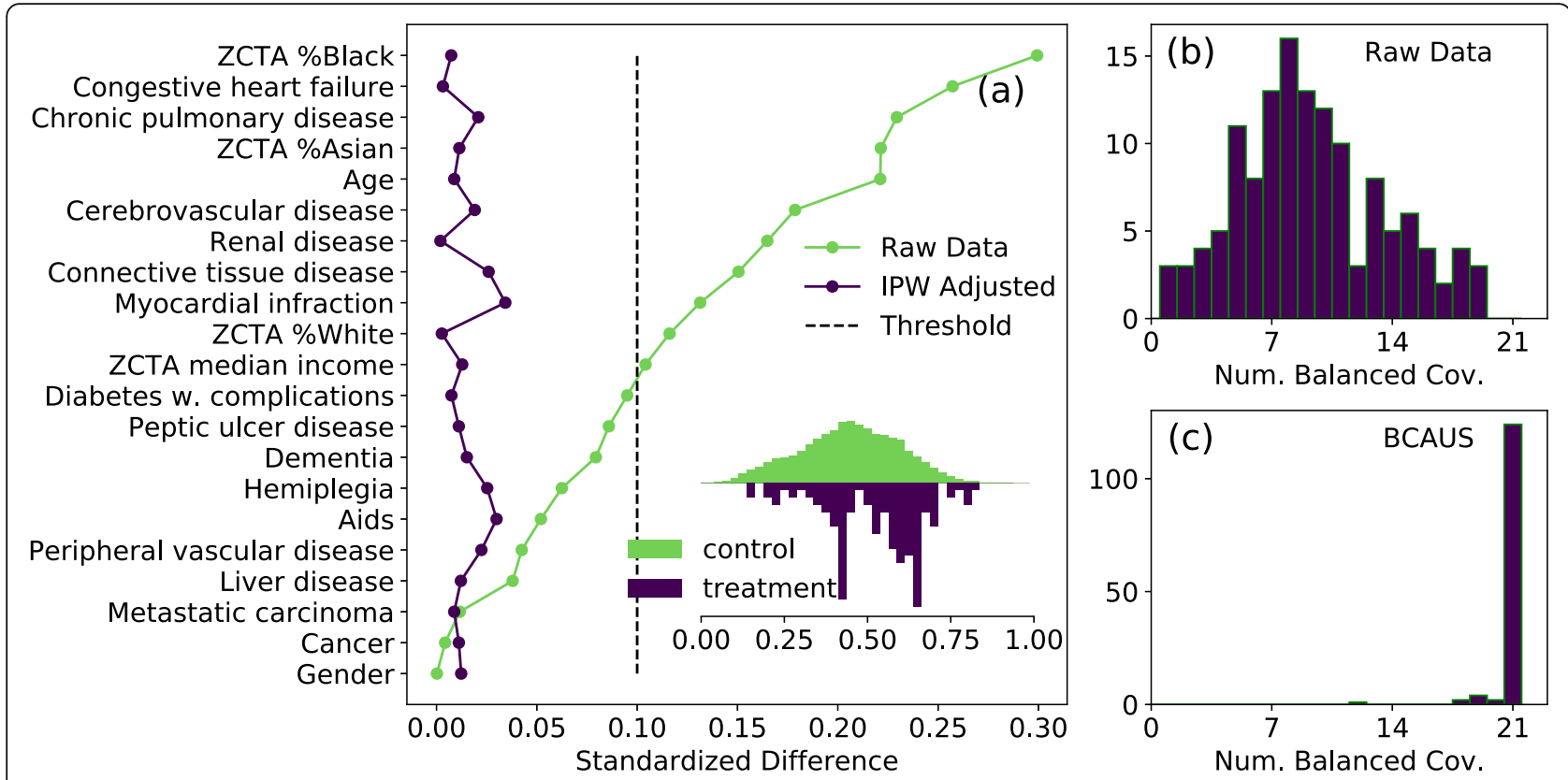

Fig. 3 Results of BCAUS on Diabetes dataset. a Standardized differences for all 21 covariates in Diabetes dataset for one arm: Treatment = Insulin + Metformin + GLP-1 Agonist + SGLT2 Inhibitor + Sulfonyurea; Control = Insulin. Cohort sizes are 125 and 44,600 respectively, implying a classimbalance ratio 1:350. Green trace is raw unadjusted data and violet trace shows data adjusted by IPW. Dashed line represents threshold at 0.1. Inset shows normalized histograms of the distribution of propensity scores i.e. output of BCAUS, for control (green) and treatment (violet) groups. ZCTA = Zip-Code Tabulation Area. $\mathbf{b}$ Histogram of number of balanced covariates (standardized difference, $\Delta<0.1$ between control and treatment) in each intervention arm prior to BCAUS training and IPW adjustment. Shown here for 133 intervention arms. c Same as (b), but after BCAUS training and adjustment. For a majority of intervention arms (124 of 133) all covariates are balanced

intervention arms tended to have very few covariates balanced prior to training and adjustment (left tail of distribution in Fig. 3(b)). For one intervention arm which had only 2 covariates balanced in the raw unadjusted data, BCAUS was able to balance only 12 covariates.

We measured covariate imbalance for all 133 intervention arms before and after IPW adjustment comparing BCAUS against two baseline models: (i) logistic regression and (ii) a neural network classifier trained only to predict treatment assignment. We chose logistic regression as a baseline because it is often used for propensity modeling in observational studies, while the neural network was chosen to emphasize that a non-linear model does not automatically guarantee covariate balance. To make the comparison fair, we used the same model architecture (number of layers, neurons etc.) for the baseline neural network model as BCAUS. Note that this can also be achieved simply by setting the hyperparameter $v$ to zero. Both baseline models were used "outof-the-box" in that no attempt was made to iteratively tweak them if covariate balance was not achieved. Results of this comparison are shown in Fig. 4. Each box plot shows the distribution of standardized differences $\Delta$ (between treatment and control arms) for a particular covariate for all 133 intervention arms. The red dashed line shows the 0.1 threshold level. The left panel shows the unadjusted data and we observe that large imbalances exist in the covariates. When weighted by IPWs from the logistic regression model or the neural network model, covariate imbalance is reduced as shown in panels (b) and (c) respectively. However, a much more dramatic reduction in imbalance is seen in the BCAUS model as shown in panel (d). This demonstrates that BCAUS is effective at balancing covariates in real world datasets with an extremely large number of intervention arms and outperforms common baselines.

We computed ATEs using propensity scores from BCAUS using IPTW for all intervention arms with 21 balanced covariates and compared it against ATE values from BART trained using the default specifications recommended by Chipman et al. [15]. Figure 5 plots BCAUS ATE values against BART ATE values for the 133 intervention arms. We observe good agreement between the two methods with a mean absolute error between the two estimates of 0.04 . While the results of the two models are comparable, it is not possible to say which one is more accurate because the ground truth ATEs for this real-world dataset are unknown. Since BART is regression based and not propensity-scorebased, we do not compare BCAUS and BART in terms of their ability to achieve covariate balance. We note 


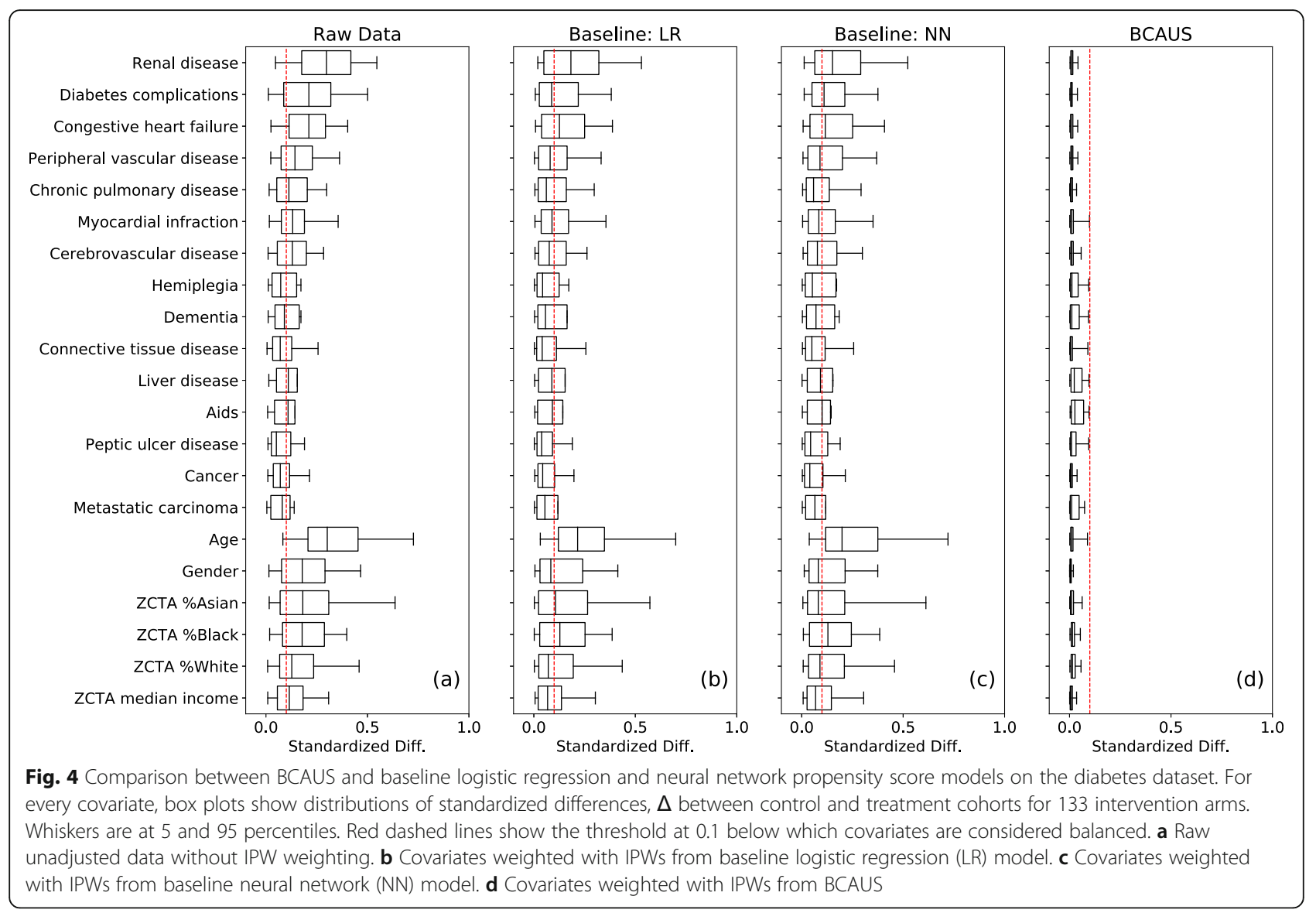

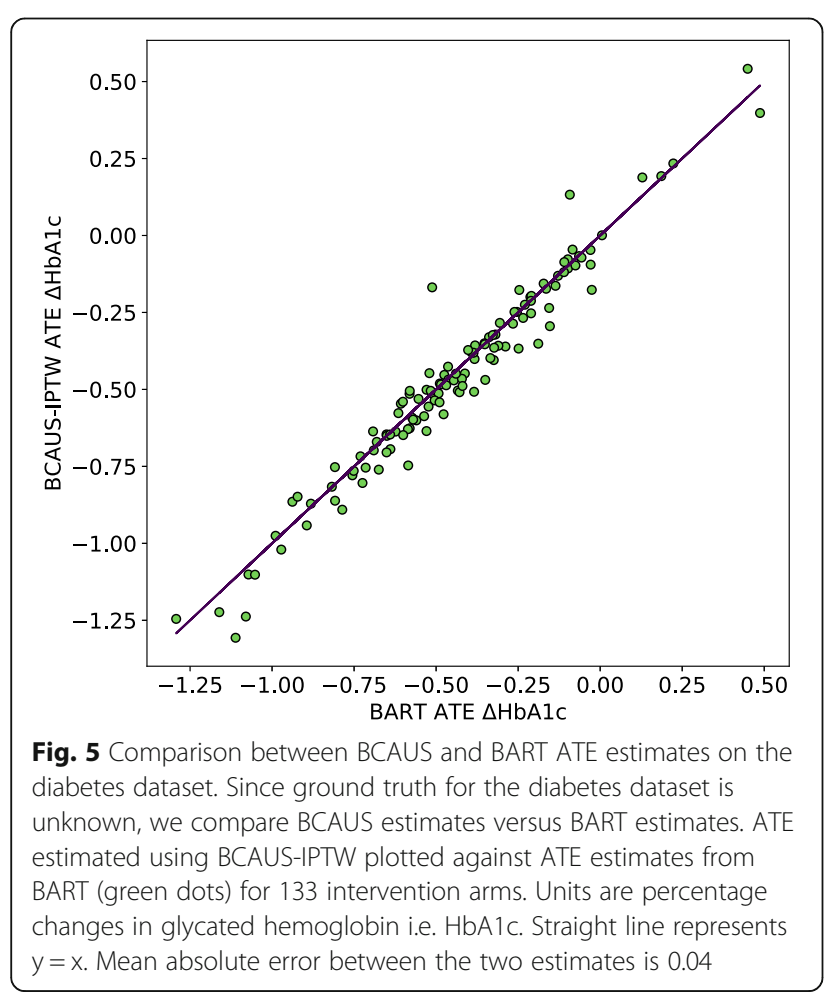

here that since BART relies on Monte Carlo sampling, we found it to be substantially slower than BCAUS with a run time of approximately $29 \mathrm{~h}$ compared to $50 \mathrm{~min}$ for BCAUS.

\section{Discussion}

The estimation of causal effects is essential in nearly every field as correct estimation empowers us to optimize decision making based on evidence. Large observational datasets that are becoming increasingly common in healthcare and medicine may have a multitude of intervention arms and require causal inference techniques that scale appropriately. While causal inference workflows that are automated are critical, it is also desirable that these methods be fast and compatible with more conventional workflows so that their results can be trusted. Many recently proposed deep-learning-based methods are highly automated and fast. BCAUS complements these algorithms and at the same time utilizes methods and diagnostics that are commonly used for observational studies in medicine.

On the semi-synthetic IHDP benchmark where ground-truth is known, BCAUS was able to correctly estimate the ATE to within an error that was comparable to other deep-learning-based methods. Of the models 
considered for comparison in Table 1 BNN, BLR, CFR and TARNet use neural networks to learn representations of the baseline covariates and train regression models on these learned representations. A comparison of the performance of these models with BART and other non-neural-network-based methods has been reported by Shalit et al. [22]. GANITE and CAVAE use generative adversarial networks and variational autoencoders respectively to learn the probability distributions of the covariates and outcomes and to estimate individual treatment effects. These methods do not generate propensity scores and cannot be used in conjunction with IPTW or DR methods. The Dragonnet method, on the other hand, models both treatment assignment as well as outcomes and is very similar to BCAUS DR. While Draggonet uses a neural network for outcome modeling, BCAUS DR, as demonstrated here, used a simple linear regressor for this task. The difference in performance between the two methods may be attributed to the more flexible modeling scheme used in Dragonnet. We also observed a difference in performance between BCAUS IPTW and BCAUS DR that suggests that despite balancing all covariates there was some residual misspecification in the propensity-score model that was corrected in the DR framework by the regression model. Setting a tighter balancing threshold (lower than 0.1) and training the network for longer may help reduce the difference between the two estimates.

On the real-world diabetes dataset involving significant covariate imbalance as well as class imbalance across hundreds of simultaneous intervention arms, BCAUS demonstrated better performance at reducing imbalance than either logistic regression or neural network propensity models. The ground-truth ATEs are unknown for this dataset, but BCAUS estimates using IPTW were comparable to BART estimates. In our runs we found BCAUS to be $\sim 30$ times faster than BART at analyzing the 133 intervention arms in the diabetes dataset.

While BCAUS was able to generate correctly specified propensity models for a majority of intervention arms in the diabetes dataset, in a small number of cases all covariates could not be balanced. The loss term $\mathcal{L}_{B I A S}$ attempts to match the first moments of IPW adjusted covariates. Supplementing this loss with terms which match higher moments [14] may ameliorate this issue. The effectiveness of the bias loss at correcting class imbalance also needs further investigation. This loss term assigns equal importance to imbalance in each covariate. If it is important to reduce imbalance in certain covariates more than in others, the mean squared error may be replaced by a weighted mean of squared errors where weights are assigned by the modeler in proportion to the relative importance of each covariate. While not explored in the present manuscript, BCAUS may also be used for time-varying confounding. In this case the propensity score output by BCAUS can be used to compute inverse propensity weights that may then be used to solve for the appropriate estimating equations of the marginal structural model under consideration. We will report on these extensions of BCAUS in future publications.

\section{Conclusion}

BCAUS can generate correctly specified propensity models for curated benchmark datasets as well as farfrom-ideal, real-world datasets. However, to get accurate estimates of the causal effect, it is essential that all potential confounders are identified for each arm of the study using subject-matter expertise and only confounding covariates are used for propensity score modeling. When used in conjunction with well-established causalinference techniques it can match the performance of recently proposed neural network methods. It scales well to cases where there are numerous intervention arms, where class imbalance is severe, and where traditional techniques of iterating between model tuning and covariate balance testing are impractical. It is our expectation that BCAUS will automate and speed up current causal inference modeling approaches in medicine and enable the design of massive multi-arm studies that were previously infeasible.

\section{Abbreviations \\ BCAUS: Balancing Covariates Automatically Using Supervision; RCT: Randomized Control Trial; FDA: Food and Drugs Administration; IPTW: Inverse Probability of Treatment Weighting; DR: Double Robust; BART: Bayesian Additive Regression Trees; ATE: Average Treatment Effect; IHDP: Infant Health and Development Program; ReLu: Rectified Linear Unit; IPW: Inverse Propensity Weight; BCE: Binary Cross Entropy; T2DM: Type-2 Diabetes Mellitus; HbA1c: Hemoglobin A1c; ICD: International Classification of Diseases; ZCTA: Zip Code Tabulation Area; GLP-1: Glucagon-Like Peptide 1; SGLT2: Sodium-Glucose Cotransporter 2; LR: Logistic Regression; NN: Neural Network}

\section{Acknowledgements}

The authors wish to thank Paula Alves, Stephanie Chong, Stefanos Giampanis, and Charlotte Knott for fruitful discussions.

\section{Authors' contributions}

C.B. developed the algorithm and the software implementation. C. B and W.S. ran the experiments. C.B., W.S., and B.N. analyzed the results and wrote the manuscript. B.N. supervised the study. The author(s) read and approved the final manuscript.

\section{Funding}

Not applicable.

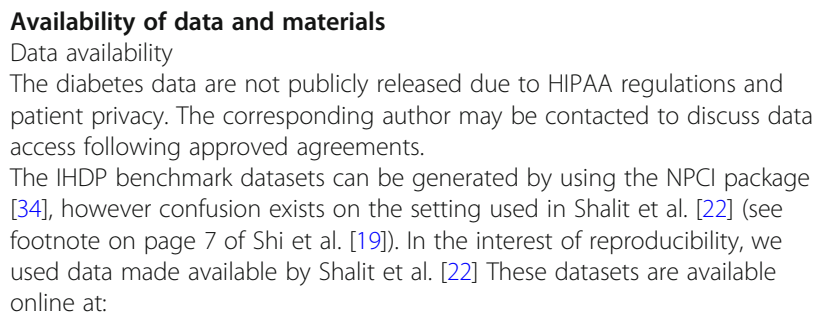


Train + CV 672 samples: http://www.fredjo.com/files/ihdp_npci_1-1000.train npz.zip

Hold-out 75 samples: http://www.fredjo.com/files/ihdp_npci_1-1000.test.npz. zip

Code availability

Code for BCAUS training and evaluation on the IHDP benchmark has been made available as Supplementary Material with the manuscript and will be hosted online at https://github.com/gstef8/bcaus_nma. The repository will maintain the code referenced here and also offer additional enhancements and extensions as they develop.

\section{Declarations}

Ethics approval and consent to participate

Not applicable.

\section{Consent for publication}

Not applicable.

\section{Competing interests}

The authors declare no competing interests.

Received: 7 May 2021 Accepted: 26 August 2021

Published online: 20 September 2021

\section{References}

1. Athey S. Beyond prediction: using big data for policy problems. Science. 2017;355(6324):483-5. https://doi.org/10.1126/science.aal4321.

2. Maathuis $\mathrm{MH}$, Colombo D, Kalisch M, Bühlmann P. Predicting causal effects in large-scale systems from observational data. Nat Methods. 2010;7(4):2478. https://doi.org/10.1038/nmeth0410-247.

3. Prosperi M, Guo Y, Sperrin M, Koopman JS, Min JS, He X, et al. Causal inference and counterfactual prediction in machine learning for actionable healthcare. Nature Machine Intelligence. 2020;2(7):369-75. https://doi.org/1 0.1038/s42256-020-0197-y.

4. Shiffrin RM. Drawing causal inference from big data. Proc Natl Acad Sci. 2016;113(27):7308-9. https://doi.org/10.1073/pnas.1608845113.

5. Varian HR. Causal inference in economics and marketing. Proc Natl Acad Sci U S A. 2016;113(27):7310-5. https://doi.org/10.1073/pnas.1510479113.

6. Munk NE, Knudsen JS, Pottegård A, Witte DR, Thomsen RW. Differences between randomized clinical trial participants and real-world Empagliflozin users and the changes in their glycated hemoglobin levels. JAMA Netw Open. 2020;3(2):e1920949. https://doi.org/10.1001/jamanetworkopen.2019.2 0949.

7. Klonoff DC. The expanding role of real-world evidence trials in health care decision making. J Diabetes Sci Technol. 2020;14(1):174-9. https://doi.org/1 $0.1177 / 1932296819832653$.

8. Rosenbaum PR, Rubin DB. The central role of the propensity score in observational studies for causal effects. Biometrika. 1983;70(1):41-55. https:// doi.org/10.1093/biomet/70.1.41

9. Rosenbaum PR. Model-based direct adjustment. J Am Stat Assoc. 1987; 82(398):387-94. https://doi.org/10.1080/01621459.1987.10478441.

10. Robins JM, Rotnitzky A, Zhao LP. Estimation of regression coefficients when some regressors are not always observed. J Am Stat Assoc. 1994;89(427): 846-66. https://doi.org/10.1080/01621459.1994.10476818.

11. Imbens GW, Rubin DB. Causal inference for statistics, social, and biomedical sciences: an introduction. Cambridge: Cambridge University Press; 2015.

12. Desai RJ, Franklin JM. Alternative approaches for confounding adjustment in observational studies using weighting based on the propensity score: a primer for practitioners. BMJ. 2019;367:I5657. https://doi.org/10.1136/bmj. 15657.

13. Hainmueller J. Entropy balancing for causal effects: A multivariate reweighting method to produce balanced samples in observational studies. Polit Anal. 2012;20(1):25-46. https://doi.org/10.1093/pan/mpr025.

14. Imai K, Ratkovic M. Covariate balancing propensity score. J R Stat Soc B Stat Methodol. 2014;76(1):243-63. https://doi.org/10.1111/rssb.12027.

15. Chipman HA, George El, McCulloch RE. BART: Bayesian additive regression trees. Ann Appl Stat. 2010;4(1):266-98. https://doi.org/10.1214/09-AOAS285.

16. Hill JL. Bayesian nonparametric modeling for causal inference. J Comput Graph Stat. 2011;20(1):217-40. https://doi.org/10.1198/jcgs.2010.08162.
17. Dorie V, Hill J, Shalit U, Scott M, Cervone D. Automated versus do-it-yourself methods for causal inference: lessons learned from a data analysis competition. Stat Sci. 2019;34(1):43-68. https://doi.org/10.1214/18-STS667.

18. LeCun Y, Bengio Y, Hinton G. Deep learning. Nature. 2015;521(7553):436-44. https://doi.org/10.1038/nature14539.

19. Shi C, Blei DM, Veitch V. Adapting neural networks for the estimation of treatment effects. arXiv preprint arXiv:1906.02120. 2019

20. Alaa AM, Weisz M, Van Der Schaar M. Deep counterfactual networks with propensity-dropout. arXiv preprint arXiv:1706.05966. 2017.

21. Johansson F, Shalit U, Sontag D. Learning representations for counterfactual inference. in International conference on machine learning; 2016.

22. Shalit U, Johansson FD, Sontag D. Estimating individual treatment effect: generalization bounds and algorithms. In: International conference on machine learning: PMLR; 2017.

23. Louizos, C., et al. Causal effect inference with deep latent-variable models. in Advances in Neural Information Processing Systems. 2017.

24. Yoon J, Jordon J, Van Der Schaar M. GANITE: Estimation of individualized treatment effects using generative adversarial nets. in International Conference on Learning Representations; 2018.

25. Yao Xl, et al. Reporting and Guidelines in Propensity Score Analysis: A Systematic Review of Cancer and Cancer Surgical Studies. JNCl. 2017;109(8): djw323.

26. Deb S, Austin PC, Tu JV, Ko DT, Mazer CD, Kiss A, et al. A review of propensity-score methods and their use in cardiovascular research. Can J Cardiol. 2016;32(2):259-65. https://doi.org/10.1016/j.cjca.2015.05.015.

27. Ali MS, Groenwold RHH, Belitser SV, Pestman WR, Hoes AW, Roes KCB, et al. Reporting of covariate selection and balance assessment in propensity score analysis is suboptimal: a systematic review. J Clin Epidemiol. 2015; 68(2):122-31. https://doi.org/10.1016/j.jclinepi.2014.08.011.

28. Austin PC. Balance diagnostics for comparing the distribution of baseline covariates between treatment groups in propensity-score matched samples. Stat Med. 2009;28(25):3083-107. https://doi.org/10.1002/sim.3697.

29. Austin PC, Stuart EA. Moving towards best practice when using inverse probability of treatment weighting (IPTW) using the propensity score to estimate causal treatment effects in observational studies. Stat Med. 2015; 34(28):3661-79. https://doi.org/10.1002/sim.6607.

30. Alaa AM, van der Schaar M. Bayesian inference of individualized treatment effects using multi-task gaussian processes. in Advances in Neural Information Processing Systems; 2017.

31. Brooks-Gunn J, Liaw F-r, Klebanov PK. Effects of early intervention on cognitive function of low birth weight preterm infants. J Pediatr. 1992; 120(3):350-9. https://doi.org/10.1016/S0022-3476(05)80896-0.

32. Charlson ME, Pompei $P$, Ales KL, MacKenzie CR. A new method of classifying prognostic comorbidity in longitudinal studies: development and validation J Chronic Dis. 1987;40(5):373-83. https://doi.org/10.1016/0021-9681(87)901 71-8.

33. United States Census Bureau. Available from: https://data.census.gov/ cedsci/.

34. Dorie, V., NPCl: Non-parametrics for Causal Inference (https://github.com/ vdorie/npci). 2016

\section{Publisher's Note}

Springer Nature remains neutral with regard to jurisdictional claims in published maps and institutional affiliations.

Ready to submit your research? Choose BMC and benefit from:

- fast, convenient online submission

- thorough peer review by experienced researchers in your field

- rapid publication on acceptance

- support for research data, including large and complex data types

- gold Open Access which fosters wider collaboration and increased citations

- maximum visibility for your research: over $100 \mathrm{M}$ website views per year

At $\mathrm{BMC}$, research is always in progress.

Learn more biomedcentral.com/submission 